\title{
DÉCEMBRE 1959
}

\section{REVUE}

\section{INTERNATIONA LE}

\author{
DE LA
}

CROIX-ROUGE

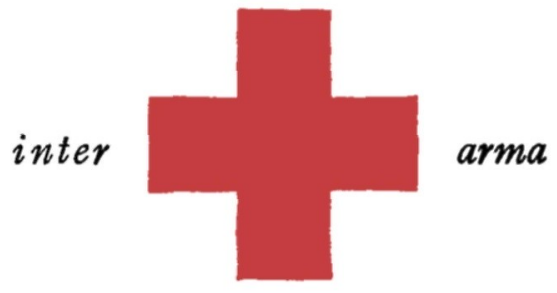

caritas

COMITE INTERNATIONAL DE LA CROIX-ROUGE GENEVE 


\title{
COMITÉ INTERNATIONAL DE LA CROIX-ROUGE
}

\section{à Genève}

\author{
Président d'honneur: M. MAX HUBER, docteur en droit, \\ ancien président de la Cour permanente de justice internationale
}

MM. LÉOPOLD BOISSIER, docteur en droit, professeur honoraire de l'Université de Genève, ancien secrétaire général de l'Union interparlementaire, président (1946) 1

JACQUES CHENEVIEERE, docteur ès lettres h.c., vice-président d'honneur (1919)

M110 LUCIE ODIER, ex-chef du Service des infirmières-visiteuses de la Section genevoise de la Croix-Rouge suisse (1930)

MM. CARL J. BURCKHARDT, docteur en philosophie, ancien ministre de Suisse en France (1933)

MARTIN BODMER, docteur en philosophie h.c. (1940)

ERNEST GLOOR, docteur en médecine (1945)

PAUL RUEGGER, ancien ministre de Suisse en Italie et en Grande-Bretagne, membre de la Cour Permanente d'Arbitrage (1948)

HENRI GUISAN, général, ancien commandant en chef de l'arnée suisse (1948)

RODOLFO OLGIATI, docteur en médecine h.c., ancien directeur du Don suisse (1949)

M"10 MARGUERITE VAN BERCHEM, ancien chef de service de l'Agence centrale des prisonniers de guerre (1951)

MM. FRÉDÉRIC SIORDET, avocat, conseiller du Comité international de la Croix-Rouge de 1943 à 1951 . vice-président (1951)

MARCEL JUNOD, docteur en médecine, délégué du Comité international de la Croix-Rouge de 1935 à 1946, vice-président (1952)

GUILI.AUME BORDIER, ingénieur dipl. E.P.F., M.B.A. Harvard, banquier (1955)

ADOLPHE FRANCESCHETTI, docteur en médecine, professeur de clinique ophtalmologique à l'Université de Genève (1958)

HANS BACHMANN, docteur en droit, secrétaire général adjoint du Comité international de la CroixRouge de 1944 à 1946 (1958)

JACQUES FREYMOND, docteur ès lettres, directeur de l'Institut universitaire de bautes études internationales, professeur à l'Université de Genève (1059)

\section{Direction:}

MM. ROGER GALLOPIN, docteur en droit, directeur exécutif

JEAN S. PICTET, docteur en droit, directeur des Affaires générales

EDOUARD de BONDELI, sous-directeur, Services financiers et administratifs

CLAUDE PILLOUD, sous-directeur, Service juridique

1 Les années indiquées dans les parenthèses désignent les dates de nomination des membres du Comité international. 


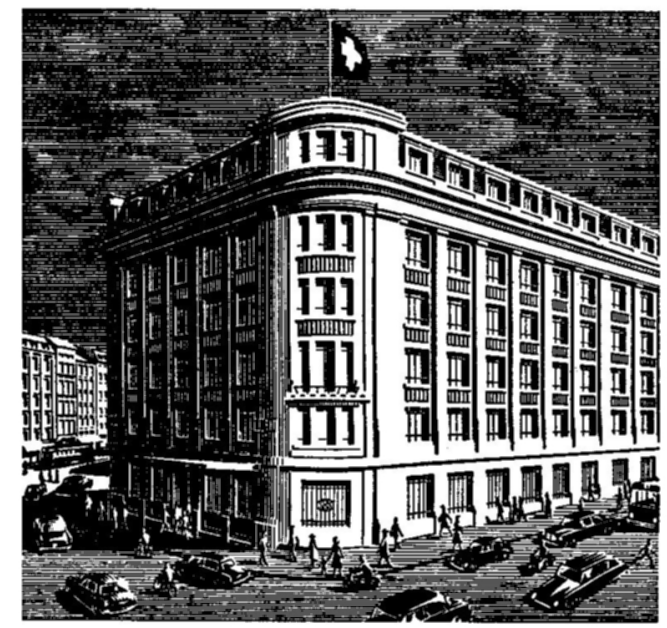

SOCIETE DE

BANQUE SUISSE SCHWEIZERISCHER BANKVEREIN SOCIETA DI BANCA SVIZZERA SWISS BANK CORPORATION

\section{GENEVE}

BALE BIENNE LA CHAUX-DE-FONDS LAUSANNE NEUCHATEL ST-GALL SCHAFFHOUSE ZURIOH LONDRES NEW-YORK

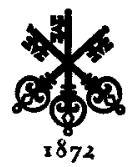

CAPITAL ET RÉSERVES FRS 293 MILLIONS 


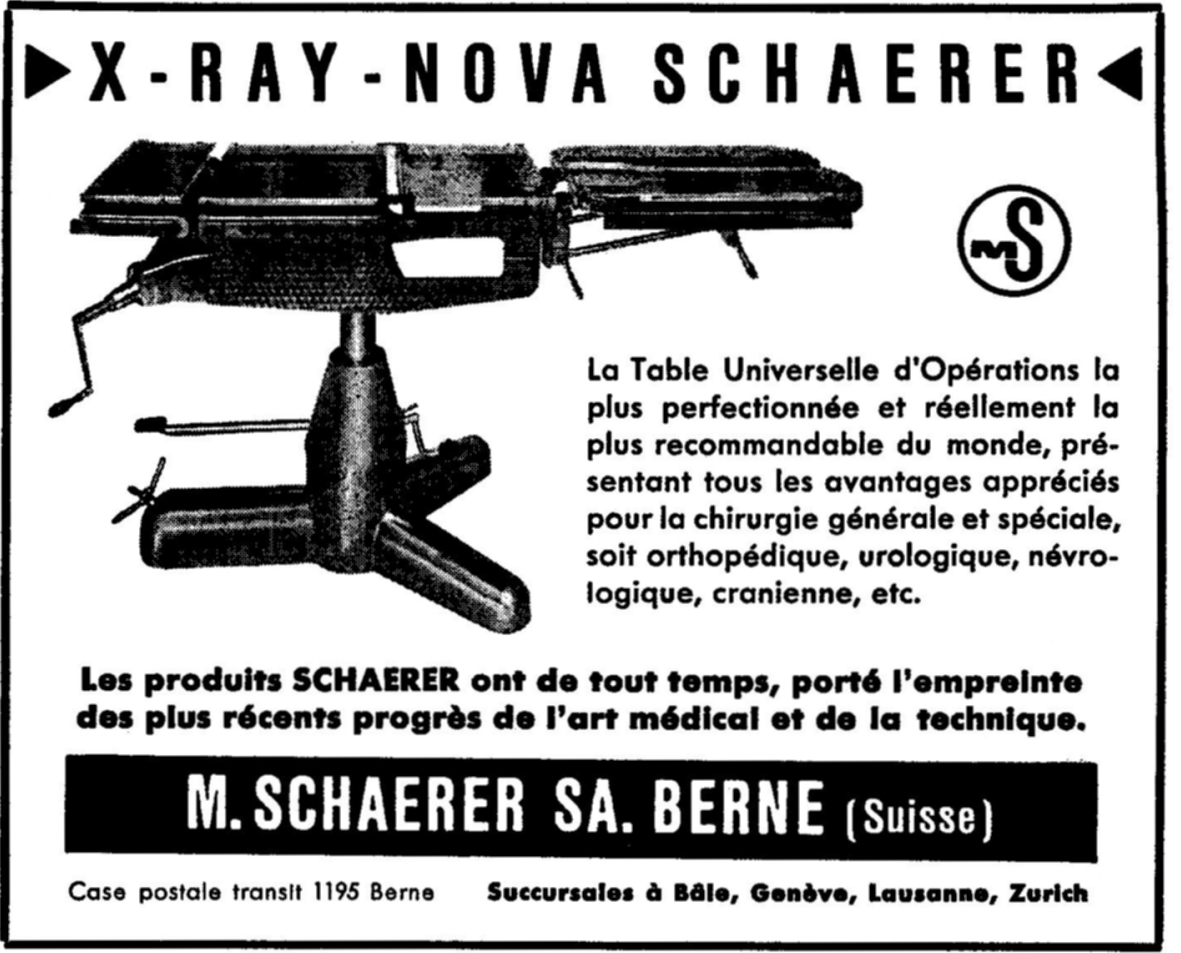

\section{SOCIETE FIDUCIAIRE ROMANDE OFOR S.A.}

9, rue d'Italie, Genève - Tél. 243293

$\downarrow$ Expertises, revisions et organisations comptables

$\uparrow$ Domiciliation et administration de sociétés

$\downarrow$ Assainissements et liquidations

- Interventions et conseils en matière fiscale 


\section{REVUE INTERNATIONALE DE LA CROIX-ROUGE}

QUARANTE ET UNIËME ANNEE - N0 492

DECEMBRE 1959

\section{SOMMAIRE}

Le centenaire de l'idée de la Croix-Rouge (F. Siordet, M. Bodmer, J. Z.) 597

\section{COMITÉ INTERNATIONAL DE LA CROIX-ROUGE}

Vice-Présidents . . . . . . . . . . . . 623

Conseil de Présidence. . . . . . . . . . . . . . . . . . . 623

Un jubilé, une distinction. . . . . . . . . . . . . . . . . 623

Le Comité international en Indonésie . . . . . . . . . . . . 624

Mission du CICR en Yougoslavie . . . . . . . . . 625

NOUVELLES DE SOCIETEÉS NATIONALES

Chili .................. 626

Liechtenstein . . . . . . . . . . . . . . . . . . 634

Suède. . . . . . . . . . . . . . . . . . . 635

Yougoslavie . . . . . . . . . . . . . 638

\section{BIBLIOGRAPHIE}

Table des matières (Année 1959) . . . . . . . . . . . . . . 647 


\section{SUPPLEMENTS DE LA REVUE}

EN LANGUE ANGLAISE

Information Meeting by the International Committee of the Red Cross (Athens, September I959). - The International Committee in Indonesia. - A mission of the ICRC in Jugoslavia. - Table of Contents. Vol. XII (I 959).

EN LANGUE ESPAGNOLE

Reunión de información del Comité Internacional de la Cruz Roja (Atenas, septiembre 1959. - El Comité Internacional en Indonesia. - Misión del CICR en Yugoeslavia. - Indice de sumarios, vol. XI (1959).

\section{EN LANGUE ALLEMANDE}

Informationssitzung des Internationalen Komitees vom Roten Kreuz (Athen, September I959). - Das Internationale Komitee in Indonesien. - Mission des IKRK in Jugoslawien. - Inhaltsverzeichnis, Band X (1959).

\section{LA}

REVUE INTERNATIONALE DE LA CROIX-ROUGE est publiée chaque mois par le Comité international de la Croix-Rouge

7, avenue de la Paix, Genève (Suisse) - Compte de chèques postaux I. 1767

Abonnement un an : Fr. 20,-; le numéro Fr. 2,-

Pour les pays suivants ; Algérie, Allemagne, Autriche, Belgique, Danemark, Finlande, France, Italie, Luxembourg, Maroc, Norvege, Pays-Bas, Portugal, Sarre, Suede, Tunisie, Vatican, on peut $s^{*}$ abonner auprès des bureaux de poste (fr. s. 20,50)

Rédaction : JEAN-G. LOSSIRR 